\title{
EXPECTATIVAS DOS ALUNOS QUANTO À IMPORTÂNCIA DA BIOQUÍMICA EM SUA CARREIRA
}

\author{
Rodrigo Cardoso de Oliveira ${ }^{1}$, Natália Fernanda Garro Monteiro ${ }^{2}$, Flávia Godoy Iano ${ }^{3}$,
} Thelma Lopes da Silva ${ }^{4}$, Marília Afonso Rabelo Buzalaf ${ }^{5}$

1. Professor Associado - rodrigocardoso@usp.br. 2. Fonoaudióloga. 3. Doutoranda do curso de Biologia Oral. 4. Especialista de Laboratório. 5. Professora Titular. Departamento de Ciências Biológicas, Faculdade de Odontologia de Bauru, Universidade de São Paulo/USP

\section{Resumo:}

O objetivo do presente trabalho foi avaliar a perspectiva dos estudantes do primeiro ano dos cursos de Odontologia e Fonoaudiologia sobre a importância do uso dos conhecimentos de bioquímica na aplicação clínica em suas futuras carreiras. Foram elaborados dois questionários simples de múltipla escolha e questões abertas sobre a importância da disciplina de bioquímica. Os questionários foram aplicados aos alunos do primeiro ano dos cursos de Odontologia e Fonoaudiologia da Faculdade de Odontologia de Bauru, Universidade de São Paulo, no começo e no final da disciplina de Bioquímica (anos de 2007 e 2008). Os estudantes responderam os questionários de forma voluntária e sem a necessidade de se identificarem. As respostas foram divididas em (questões de múltipla escolha) e avaliadas quanto ao conteúdo escrito (questões abertas), indicando a opinião do aluno em cada questão. Cento e quinze estudantes responderam o primeiro questionário e cento e trinta e nove estudantes responderam o segundo. Na maioria das questões de múltipla escolha, a opinião predominante foi que a disciplina de bioquímica é importante para sua futura carreira $(59,0 \%)$ bem como para sua rotina clínica $(51,1 \%)$. Podemos concluir que os estudantes estão conscientes da importância dos conhecimentos adquiridos em Bioquímica para sua futura carreira, embora ainda não sejam capazes de definir com clareza a forma como esses conhecimentos serão aplicados.

Palavras-Chave: Bioquímica, conhecimento, área da saúde.

\begin{abstract}
:
The purpose of the present study was to evaluate the perspective of students in the first year of Dental and Speech, Language and Hearing Sciences School regarding the use of knowledge in Biochemistry for clinical applications in their future career. Two simple questionnaires were elaborated consisting of multiple choice and open questions regarding the Biochemistry discipline. The questionnaires were given to first-year Dental and Speech, Language and Hearing Sciences students at Bauru Dental School, University of São Paulo, at the beginning and the end of their Biochemistry course (2007 and 2008 years). The students answered the questions voluntarily and in an unidentified format. The answers were then divided (multiple choice questions) and the written contents (open questions) evaluated, indicating the students' opinion with regards to each question. One hundred and fifteen students answered the first questionnaire and one hundred and thirty nine answered the second one. In the great majority of the multiple choice questions, the predominant opinion was that the Biochemistry discipline is important for their future careers $(59.0 \%)$ as well as for their routine clinical setting $(51.1 \%)$. We may conclude that students are aware of the importance of the acquired knowledge in Biochemistry for their future career although are not yet capable of defining how such knowledge will be applied.
\end{abstract}

Key-words: Biochemistry, knowledge, health area. 


\section{INTRODUÇÃO}

A chamada "Era do Conhecimento" introduziu algumas exigências em diversas áreas profissionais. Entre as habilidades descritas como essenciais para o futuro profissional estão: habilidades interpessoais (boa comunicação, capacidade de trabalhar em equipe e sintonia com parceiros/clientes), pensamento crítico, aprendizagem contínua [1, 2] e construção de conhecimento [3]. De acordo com a Association for Dental Education in Europe (ADEE), alguns desses requisitos são muito importantes para o cirurgião-dentista, tais como conhecimento e compreensão da importância da formação científica na odontologia (incluindo ciências básicas e biomédicas), entender os mecanismos de doenças incluindo infecção, inflamação, desordens do sistema imune, degeneração, neoplasias, alterações genéticas e metabólicas. Além de compreender a ciência dos materiais dentários [4]. Boa parte dessas características pode ser preenchida com a aprendizagem de conceitos ministrados nas disciplinas básicas, como, histologia, anatomia, fisiologia, farmacologia, bioquímica e outras.

O ensino das disciplinas básicas, nos cursos de Odontologia e Fonoaudiologia, têm por objetivo formar um profissional embasado para realizar procedimentos de diagnóstico, tratamento e prognóstico de pacientes [5]. Em outras palavras, as disciplinas de ciências básicas ministradas no inicio dos cursos de Odontologia e Fonoaudiologia devem proporcionar base sólida de conhecimentos para ser aplicada na rotina diária do profissional [6]. Tais conhecimentos não teriam nenhum valor real se não fossem usados na rotina profissional [7]. Por isso, especificamente o ensino de bioquímica, tem sido uma preocupação de diversos docentes [8], tendo em vista que é utilizada como uma ferramenta nos procedimentos de diagnóstico, tratamento e prognóstico por diversos profissionais da área da saúde [9]. Entretanto, em geral, as ciências básicas apresentam certo grau de rejeição por parte dos alunos e até mesmo professores de outras disciplinas $[10,11]$. O sistema de currículo, no Brasil, parece influenciar este fenômeno devido a sua estrutura fragmentada de disciplinas isoladas e desvinculadas da realidade profissional, hipertrófico em conteúdo e tem como objetivo uma formação técnica e específica, não formando profissionais que atendam as necessidades de saúde da sociedade [12-16]. Em particular, a disciplina de Bioquímica é apresentada nos programas mais tradicionais como uma disciplina organizada e coerente, porém muitas vezes definida pelos alunos como uma coleção de estruturas químicas e reações, dificilmente assimiladas e desintegradas de sua prática profissional [12-15]. No entanto, essa falta de interesse pela bioquímica parece ter diminuído desde os recentes avanços no campo das ciências, tais como o Projeto Genoma Humano e o uso de células-tronco, além disso, o próprio mercado de trabalho tem cobrado um maior nível de compreensão e conhecimento de ciências básicas [17].

Outro aspecto a ser levado em consideração é que a importância das disciplinas das ciências básicas pode ser muitas vezes negligenciada devido a razões menores. Um bom exemplo disso é a ansiedade do próprio aluno para a prática de situações clínicas com formação apenas de manobras técnicas e repetitivas, sem entender o contexto clínico do paciente e o conjunto de todo o caso clínico: diagnóstico-tratamento-prognóstico. Essa ansiedade em "pular etapas" pode levar o aluno a diminuir ou, na pior das hipóteses, até mesmo negligenciar o conhecimento de ciências básicas [18, 19], incluindo Bioquímica, levando assim aos problemas durante a prática clínica.

Portanto, o objetivo do presente estudo foi avaliar a perspectiva dos estudantes de Odontologia e Fonoaudiologia, do primeiro ano, sobre a importância dos conhecimentos em bioquímica para a sua futura carreira.

\section{MATERIAL E MÉTODO}

Foram aplicados dois questionários simples compostos de questões abertas e de múltipla escolha, sobre a importância e aplicabilidade da disciplina de bioquímica no 
cotidiano clínico, seguindo o modelo proposto por Haddad et al., 1993 [20]. Este estudo foi aprovado pelo Comitê de Ética em Pesquisa da Faculdade de Odontologia de Bauru, Universidade de São Paulo (protocolo $n^{\circ}$ 002/2007). A participação dos estudantes foi inteiramente voluntária e o termo de consentimento foi obtido de todos os participantes. Os questionários foram aplicados aos alunos do primeiro ano do curso de graduação em Odontologia e Fonoaudiologia, no inicio (Fase 1) e final (Fase 2) da disciplina de bioquímica nos anos de 2007 e 2008. Os estudantes responderam os questionários sem a necessidade de se identificarem para não sofrerem qualquer tipo de inibição. Um total de 115 estudantes respondeu o primeiro questionário e 139 responderam o segundo questionário.

Após a coleta dos questionários preenchidos as respostas foram divididas segundo suas alternativas escolhidas (questões de múltipla escolha) e avaliadas quanto ao conteúdo escrito (questões abertas), indicando a opinião do aluno em relação a cada questão.

Fase 1: Questionário aplicado aos alunos do curso de Odontologia e Fonoaudiologia do primeiro ano da graduação (2007 e 2008), antes da primeira aula da disciplina de Bioquímica.

\section{UNIVERSIDADE DE SÃO PAULO FACULDADE DE ODONTOLOGIA DE BAURU DEPARTAMENTO DE CIÊNCIAS BIOLÓGICAS DISCIPLINA DE BIOQUÍMICA}

Caro aluno,

Solicitamos sua colaboração respondendo este questionário, através do qual você terá oportunidade de exteriorizar sua opinião referente à disciplina de Bioquímica.

A sinceridade em suas respostas nos é muito valiosa, pois elas fornecerão subsídios para reestruturarmos a disciplina em alguns aspectos. A sua informação é anônima e não há necessidade de assinar.

Obrigado pela sua colaboração.

Docentes de Bioquímica

\section{1) 0 que você espera aprender na disciplina de Bioquímica ministrada?}

\section{2) Em sua opinião, o curso de bioquímica ministrado terá relevância na sua formação profissional?}
a) $\operatorname{Sim}$
b) Apenas em parte
c) Muito pouco
d) Nenhuma

Fase 2: Questionário aplicado aos alunos do curso de Fonoaudiologia do primeiro ano da graduação (2007 e 2008), após a última aula da disciplina de Bioquímica. 


\section{UNIVERSIDADE DE SÃO PAULO \\ FACULDADE DE ODONTOLOGIA DE BAURU \\ DEPARTAMENTO DE CIÊNCIAS BIOLÓGICAS \\ DISCIPLINA DE BIOQUÍMICA}

Caro aluno,

Solicitamos sua colaboração respondendo este questionário, através do qual você terá oportunidade de exteriorizar sua opinião referente à disciplina de Bioquímica.

A sinceridade em suas respostas nos é muito valiosa, pois elas fornecerão subsídios para reestruturarmos a disciplina em alguns aspectos. A sua informação é anônima e não há necessidade de assinar.

Obrigado pela sua colaboração.

Docentes de Bioquímica

1) Você julga que a metodologia de ensino vivenciada em Bioquímica propicia o desenvolvimento de integração entre teoria e prática?
a) Sim
b) Apenas em parte
c) Muito pouco
d) Nenhum

2) A metodologia de ensino vivenciada em Bioquímica propicia a participação ativa no seu processo de aprendizagem?
a) Sim
b) Apenas em parte
c) Muito pouco
d) Nenhuma

3) Em sua opinião, o curso de bioquímica ministrado terá relevância na sua formação profissional?
a) Sim
b) Apenas em parte
c) Muito pouco
d) Nenhum

4) O uso do conteúdo do curso de bioquímica parece ser importante no cotidiano clínico?
a) $\operatorname{Sim}$
b) Apenas alguns
c) Nenhum deles
d) Não tenho tempo suficiente de curso para responder

5) Qual(is) exemplo(s) você poderia citar sobre aplicação de um conhecimento de bioquímica numa situação clínica? 


\section{RESULTADOS}

Em relação ao questionário aplicado aos alunos do primeiro ano, a maioria dos alunos respondeu que espera aprender fenômenos que acontecem no organismo humano, tais como processos biológicos e químicos (primeira questão: "Eu espero aprender mais detalhes e compreender melhor processo químicos e biológicos do organismo humano").

No primeiro questionário, $80 \%$ dos alunos responderam que o conteúdo do curso de bioquímica será usado em sua futura profissão. Por outro lado, $20 \%$ dos estudantes acreditam que a disciplina de bioquímica terá uma relevância menor em sua atuação profissional futura (Tabela 1 ).

Tabela 1 - Distribuição da opinião dos alunos do primeiro ano dos cursos de Odontologia e Fonoaudiologia (anos de 2007 e 2008). Segunda questão do Primeiro questionário.

\section{Alternativas assinaladas}

\section{Questão}

"Na sua opinião, o curso de bioquímica ministrado terá importância na sua atuação profissional?"

\begin{tabular}{|c|c|c|c|c|}
\hline Sim & $\begin{array}{l}\text { Apenas } \\
\text { em parte }\end{array}$ & $\begin{array}{l}\text { Muito } \\
\text { pouco }\end{array}$ & Não & Total \\
\hline $\begin{array}{c}92^{a} \\
(80 \%)^{b}\end{array}$ & $\begin{array}{c}19 \\
(16,5 \%)\end{array}$ & $\begin{array}{c}4 \\
(3,5 \%)\end{array}$ & $\begin{array}{c}0 \\
(0 \%)\end{array}$ & $\begin{array}{c}115 \\
(100 \%)\end{array}$ \\
\hline
\end{tabular}

a: Número de alunos

b: Porcentagem de alunos (\%)

No questionário aplicado no final do curso de bioquímica, a opinião dos alunos para a questão sobre a integração entre teoria e prática, cerca de 49,7\% responderam que houve uma boa integração, ao passo que 36,7\% julgaram essa relação regular (Tabela 2). Sobre a metodologia de ensino vivenciada na disciplina de bioquímica, somente 49,7\% responderam que a mesma proporciona uma participação ativa nesse processo de aprendizado, por outro lado, 36,0\% dos alunos julgaram essa participação regular (Tabela 2).

$\mathrm{Na}$ questão sobre as expectativas do curso, a maioria dos estudantes $(85,6 \%)$ acredita no uso do conteúdo da disciplina na prática clínica. Aproximadamente $15 \%$ dos estudantes não acreditam que o conteúdo aprendido no curso de bioquímica terá utilidade na sua rotina profissional (Tabelas 2 e 3). Com relação ao exemplo dado pelos alunos, o uso do flúor e seus mecanismos de ação: "Os efeitos do flúor, a etiologia da cárie e da capacidade tampão da saliva" (última pergunta) foi o exemplo mais citado. 
Tabela 2 - Distribuição da opinião dos alunos de Odontologia e Fonoaudiologia do primeiro ano (anos 2007 e 2008). Primeira, segunda e terceira questões do Segundo questionário.

\section{Alternativas assinaladas}

\section{Questões}

Sim $\begin{gathered}\text { Apenas } \\ \text { em parte }\end{gathered} \quad \begin{gathered}\text { Muito } \\ \text { pouco }\end{gathered}$ Não $\quad$ Total

"Você acha que a metodologia de ensino usada em bioquímica promove a integração entre teoria e prática?"

$\begin{array}{ccccc}69^{\mathrm{a}} & 51 & 17 & 2 & 139 \\ (49,7 \%)^{\mathrm{b}} & (36,7 \%) & (12,2 \%) & (1,4 \%) & (100 \%)\end{array}$

"A metodologia de ensino vivenciada na bioquímica propicia uma participação ativa no processo de 6950 50 18
$(12,9 \%)$ 2
$(1,4 \%)$ 139 aprendizagem?"

"Em sua opinião, o curso de bioquímica é relevante para sua profissão?"

82 $(59,0 \%)$
37

$(26,6 \%)$
17

$(12,2 \%)$
139 (100\%)

a: Número de alunos

b: Porcentagem de alunos (\%)

Tabela 3- Distribuição da opinião dos alunos de Odontologia e Fonoaudiologia do primeiro ano (anos de 2007 e 2008). Quarta questão do Segundo questionário.

\begin{tabular}{|c|c|c|c|c|c|}
\hline \multicolumn{6}{|c|}{ Alternativas assinaladas } \\
\hline Questão & Sim & $\begin{array}{c}\text { Somente } \\
\text { alguns }\end{array}$ & Nenhum & $\begin{array}{l}\text { Não tenho } \\
\text { tempo de } \\
\text { curso } \\
\text { suficiente } \\
\text { para } \\
\text { responder }\end{array}$ & Total \\
\hline $\begin{array}{l}\text { acredita que o } \\
\text { lo da disciplina de } \\
\text { química será } \\
\text { oortante para } \\
\text { cação clínica?" }\end{array}$ & $\begin{array}{c}71^{a} \\
(51,1 \%)^{b}\end{array}$ & $\begin{array}{c}51 \\
(36,7 \%)\end{array}$ & $\begin{array}{c}6 \\
(4,3 \%)\end{array}$ & $\begin{array}{c}11 \\
(7,9 \%)\end{array}$ & $\begin{array}{c}139 \\
(100 \%)\end{array}$ \\
\hline
\end{tabular}

a: Número de alunos

b: Porcentagem de alunos (\%) 


\section{DISCUSSÃO}

Os recém ingressados estudantes de Odontologia e Fonoaudiologia estão, geralmente, em busca de formação técnica imediata, em vez de um conhecimento mais profundo das ciências básicas [10]. A maioria dos alunos, no presente trabalho, tinha aproximadamente 19 anos (dados não mostrados). De modo geral esses alunos são motivados a cursar a Faculdade (curso da área da saúde) por motivos: financeiro, profissional, vocação e outros [21], variando de acordo com o status social e gênero, entre outros fatores [22]. Daí, a necessidade de conhecer o perfil de cada aluno e as expectativas a respeito do curso, na tentativa de melhorar o processo de ensino e aprendizagem [14].

É importante considerar a heterogeneidade dos alunos que ingressam nas universidades do Brasil, principalmente devido ao sistema de ensino adotado no país [15], gerando a necessidade de adequação em determinadas disciplinas e também para a eficácia dos docentes no processo de ensino-aprendizagem. Nesse contexto, o presente trabalho demonstrou uma visão um pouco distorcida dos alunos (ou não, dependendo do projeto pedagógico) sobre a disciplina de Bioquímica (somente conceitos químicos e biológicos desconectados).

Dentro do processo de ensino-aprendizagem, os professores são desafiados a promover e despertar o interesse dos alunos, motivando-os na busca de novos conhecimentos e estimulando sua curiosidade científica. Não obstante, alguns fatores parecem aumentar o desafio, como para as disciplinas de ciências básicas. Um desses fatores é a estrutura tradicional dos currículos utilizados: fragmentado em disciplinas isoladas, desconectadas da realidade da profissão e com conteúdo hipertrófica, visando a uma formação técnica e não para suprir as necessidades básicas da profissão e da sociedade em geral [12]. Este relato da literatura pode ser confirmado pela discrepância dos nossos resultados obtidos na questão da importância da disciplina de Bioquímica (Tabela 3) e as respostas de aplicação do conhecimento na atividade clínica. Esse processo de desvinculação entre as disciplinas básicas e profissionalizantes (falta de integração entre professores e temas ministrados) compromete o entendimento do aluno, e por fim o uso dos conhecimentos básicos aplicados em clínica. Uma vez que a integração entre os próprios professores não acontece sempre.

Devido a essa desconexão entre as ciências básicas e clínicas, o que realmente fica comprometida é o conhecimento do aluno e outras habilidades profissionais [15]. Nossos resultados são semelhantes aos de outros trabalhos, que de acordo com as opiniões dos alunos, o método mais eficiente de aprendizagem vem da combinação de aulas teóricas e práticas $[12,23]$.

No que diz respeito ao interesse dos alunos para a aprendizagem e valorização da disciplina de Bioquímica em suas carreiras futuras, os resultados aqui foram muito semelhantes a outros estudos anteriores $[15,17]$, em que os alunos realmente acreditam que a bioquímica tem um lugar importante na sua rotina profissional.

Como descrito neste estudo, e especificamente no Brasil, observamos uma possível mudança no perfil do estudante do primeiro ano de Odontologia e Fonoaudiologia, como a sua percepção da importância e aplicabilidade de bioquímica na sua profissão. Isto deveria ser adotado pelos professores como uma avaliação contínua do plano de curso proposto bem como de metodologia de ensino utilizada [15]. Cabe aos membros do corpo docente identificar o perfil dos estudantes e, portanto, estabelecer um melhor processo de ensino, além disso, reforçam a necessidade de contínuo "feedback" dos alunos com relação à dinâmica do processo de ensino.

O desenvolvimento das disciplinas foi aprovado pela maioria dos alunos, no entanto, alguns pontos como a integração das aulas teóricas e práticas parecem ter espaço para melhorias. Podemos concluir que os alunos de Odontologia e Fonoaudiologia estão conscientes da importância dos conhecimentos adquiridos em Bioquímica para sua futura carreira profissional, embora ainda não são capazes de definir a forma como esses conhecimentos serão aplicados. 


\section{Agradecimentos}

Aos alunos dos cursos de Odontologia e Fonoaudiologia da FOB-USP, pela colaboração valiosa e espontânea nesta pesquisa.

\section{REFERÊNCIAS BIBLIOGRÁFICAS}

[1] W.D. Hendricson, S.C. Andrieu, G. Chadwick, J.E Chmar, J.R Cole, M.C George (2006) Educational Strategies Associated with Development of Problem-Solving, Critical Thinking, and Self-Directed Learning, J Dent Educ, 70, 925-36.

[2] A.D. Bullock, C.R. Belfield, S. Butterfield, P.M. Ribbins, J.W. Frame (1999) Continuing education courses in dentistry: assessing impact on practice, Med Educ, 33, 484-488.

[3] M Mylopoulos \& M. Scardamalia (2008) Doctors' perspectives on their innovations in daily practice: implications for knowledge building in health care, Med Educ, 42, 975981.

[4] J.M. Plasschaert, W.P. Holbrook, E. Delap, C Martinez, A.D. Walmsley (2005) Profile and competences for the European dentist, Eur J Dent Educ, 9, 98-107.

[5] A.J. Formicola (1986) Preprofessional preparation for the basic science curriculum, ] Dent Educ, 50, 458-461.

[6] R.M. Saunders (1996) The candidate of the future, J Dent Educ, 60, 411-415.

[7] C.M.D. Wannmacher (2001) Teaching biochemistry for future physicians (in Portuguese), RBEBBM: Available at: http://sbbq.iq.usp.br/revista/artigo.php?artigoid=1 accessed on 18 December 2008.

[8] D.K. Yokaichiya, E. Galembeck, B.B. Torres (2001) Expectations and Interest in Distance Education in Biochemistry Classes (in Portuguese), RBEBBM: Available at: http://sbbq.iq.usp.br/revista/artigo.php?artigoid=38 accessed on 17 December 2008.

[9] J.R. Davies, G. Warfvinge (2003) Basic biology integration - the Malmo model, (Abstract) Eur J Dent Educ, 7, 94.

[10] W.A. Welker (1992) The dental curriculum: is relevant to dental practice?, J. Prosthodont, $1,47-50$.

[11] B.J. Baum (2003) Can biomedical science be made relevant in dental education? A North American perspective, Eur J Dent Educ, 7, 49-55.

[12] L.H.M. Vargas (2001) Biochemistry and Problem Based Learning (in Portuguese), RBEBBM: http://www.bdc.ib.unicamp.br/rbebbm/visualizar material.php?id material=93 accessed on 10 December 2008.

[13] A.M. Iacopino, D.P. Lynch, T. Taft (2004) Preserving the Pipeline: A Model Dental Curriculum for Research Non-Intensive Institutions, J Dent Educ, 68, 44-49.

[14] J. Kieser, P. Herbison, T. Harland (2005) The influence of context on students' approaches to learning: a case study, Eur J Dent Educ, 9, 150-156.

[15] P.F. Beckhauser, E.M. Almeida, A.L.B. Zeni (2006) The Students Universe and the Biochemistry Teaching (in Portuguese), RBEBBM: Available at: http://www.bdc.ib.unicamp.br/rbebbm/visualizar_material.php?id_material $=252$ accessed on 10 December 2008. 
[16] M. Pyle, S.C. Andrieu, G. Chadwick, J.E. Chmar, J.R. Cole, M.C.I. George (2006) The Case for Change in Dental Education, J Dent Educ, 70, 921-924.

[17] R.C. Oliveira, F.G. Iano, T.L. Silva, M.A.R. Buzalaf (2007) Perception of the Dentistry Students of a Brazilian University in Relation to the Importance of Disciplines of Biochemistry (in Portuguese), RBEBBM: Available at: http://www.bdc.ib.unicamp.br/rbebbm/visualizar_material.php?id_material=346 accessed on 11 December 2008.

[18] B.J. Baum (1997) The absence of a culture of science in dental education, Eur J Dent Educ, 1, 2-5.

[19] D.A. Nash (1998) 'And the band played on . . .', ] Dent Educ, 62, 964-974.

[20] M.C.L. Haddad, M.T.O. Vannuchi, O.C. Takahashi, S.A. Hirazawa, I.G. Rodrigues, B.R. Cordeiro (1993) Medical-surgical nursing: a new approach to teaching and evaluation by student, Rev Latino-Am. Enfermagem, 1, 97-112.

[21] E. Bernabé, J.L. Icaza, E.K. Delgado-Angulo (2006) Reasons for choosing dentistry as a career: a study involving male and female first-year students in Peru, Eur J Dent Educ, 10, 236-241.

[22] M. Jover, D. Doudoux, E. Deveaux (2006) Representations of the dental surgery profession and the motivations given by second-year French students for applying for dental surgery, Eur J Dent Educ,10, 2-9.

[23] D.K. Kassebaum, W.D. Hendricson, T. Taft, K. Haden (2004) The Dental Curriculum at North American Dental Institutions in 2002-03: A Survey of Current Structure, Recent Innovations, and Planned Changes, J Dent Educ, 68, 914-931. 